\title{
Magnetically-charged black branes and viscosity/entropy ratios
}

\author{
Hai-Shan Liu, ${ }^{a, b}$ H. Lü ${ }^{c}$ and C.N. Pope $e^{b, d}$ \\ ${ }^{a}$ Institute for Advanced Physics 8 Mathematics, \\ Zhejiang University of Technology, Hangzhou 310023, China \\ ${ }^{b}$ George P. \& Cynthia Woods Mitchell Institute for Fundamental Physics and Astronomy, \\ Texas A\&M University, College Station, TX 7r843, U.S.A. \\ ${ }^{c}$ Department of Physics, Beijing Normal University, \\ Beijing 100875, China \\ ${ }^{d}$ DAMTP, Centre for Mathematical Sciences, \\ Cambridge University, Wilberforce Road, Cambridge CB3 OWA, U.K. \\ E-mail: hsliu.zju@gmail.com, mrhonglu@gmail.com, pope@physics.tamu.edu
}

ABSTRACT: We consider asymptotically-AdS $n$-dimensional black brane solutions in a theory of gravity coupled to a set of $N p$-form field strengths, in which the field strengths carry magnetic charges. For appropriately chosen charges, the metrics are isotropic in the $(n-2)$ transverse directions. However, in general the field strength configurations break the full Euclidean symmetry of the $(n-2)$-dimensional transverse space, and the shear viscosity tensor in the dual theory is no longer isotropic. We study the linearised equations for transverse traceless metric perturbations in these backgrounds, and by employing the Kubo formula we obtain expressions for the ratios $\eta / S$ of the shear viscosity components divided by the entropy density. We find that the KSS bound on the ratios $\eta / S$ is generally violated in these solutions. We also extend the discussion by including a dilatonic scalar field in the theory, leading to solutions that are asymptotically Lifshitz with hyperscaling violation.

KEYWORDS: Gauge-gravity correspondence, Holography and condensed matter physics $(\mathrm{AdS} / \mathrm{CMT})$

ARXIV EPRINT: 1602.07712 


\section{Contents}

1 Introduction 1

2 AdS planar black holes with magnetic charges 3

3 Transverse, traceless perturbations 5

4 Viscosity/entropy ratio $\quad 8$

4.1 A general discussion $\quad 9$

$\begin{array}{lr}4.2 \text { The special case } \mu=0 & 10\end{array}$

4.3 Small $\alpha$ (large $T$ ) expansion $\quad 11$

4.4 Low temperature expansion 12

5 Conclusions 13

A Lifshitz black holes with hyperscaling violation $\quad 14$

\section{Introduction}

A class of static magnetically-charged planar black hole solutions of the Einstein-Maxwell theory with a cosmological constant in an even spacetime dimension $n$ was constructed in [1]. In these solutions, the Maxwell field strength takes the form $F=\alpha\left(d x_{1} \wedge d x_{2}+\right.$ $\left.d x_{3} \wedge d x_{4}+\cdots\right)$, where $x_{i}$ are the coordinates on the Euclidean $(n-2)$-dimensional transverse space of the planar black hole. In the case of $n=4$ dimensions the solution is nothing but the standard magnetically-charged Reissner-Nordström black hole with a planar (or toroidal) horizon geometry. In even dimensions $n \geq 6$ the solutions are again asymptotic to AdS spacetime, but the magnetic charge contribution in the $g_{t t}$ and $g^{r r}$ metric functions, proportional to $1 / r^{2}$, now falls off more slowly than the black hole mass term, which falls like $1 / r^{n-3}$. This has significant implications for the properties of the boundary field theory related to the bulk theory via the AdS/CFT correspondence.

In this paper, we shall generalise the discussion in [1], by considering an $n$-dimensional theory comprising a set of $N$ form fields coupled to Einstein gravity with a (negative) cosmological constant, possibly with the addition of a dilatonic scalar as well. A subclass of such theories that we shall initially focus on comprises $N p$-form field strengths coupled to gravity with a cosmological constant. We then study the asymptotically AdS static planar black hole solutions that arise when the form fields carry magnetic charges. A particular case within this class of theories and solutions is when $p=1$, meaning that the corresponding 1-form field strengths are simply the gradients of a set of $N$ axionic scalar fields. The black hole solutions in this theory were constructed in [2], and were studied 
recently in [3], where properties such as the viscosity of the fluid described by the boundary CFT were investigated in detail.

The AdS/CFT correspondence provides a new tool to study the dynamics of stronglycoupled gauge field theories [4-7], and one of the most celebrated results is the universality, within a wide class of theories, of the ratio of shear viscosity to entropy density, namely

$$
\frac{\eta}{S}=\frac{1}{4 \pi}
$$

which was discovered, and further proposed as a lower bound, by Kovtun, Son and Starinets (KSS) [8-11]. Recently, another viewpoint was highlighted, in which (1.1) is interpreted as a holographic boundary dual to a generalized Smarr relation for the bulk gravitational backgrounds $[12,13]$.

It has been known for a while that the equality, and the KSS bound, can be violated under certain circumstances, notably in two different situations. The first of these is if the theory involves higher-derivative gravity (see e.g. [14-17],) and the second is if the gravitational background has an anisotropic transverse subspace (see e.g. [18-25]. ${ }^{1}$ ) Recently, it was observed in $[3,28,29]$ that there is a third way to violate the bound, in a theory with conventional two-derivative gravity whose gravitational background is isotropic, but with $(n-2)$ scalar matter fields having linear dependence on the $(n-2)$-dimensional transverse coordinates.

The solutions that we study in this paper provide generalisations of those that were considered in [3], and we find that again the viscosities in these systems can also violate the KSS bound. There are significant differences between the case of 1-form field strengths, as discussed in [3], and the cases with higher-degree forms. Principally, these differences arise because now, unlike in the case of $p=1$, the isotropy of the transverse space is broken by field strengths in the background solution, and as a result different transverse traceless metric fluctuations can be associated with boundary operators yielding different shear viscosities.

To be more precise, in the generic situation with an anistropic background, the shear viscosity is characterised by a four-index tensor $\eta_{i j, k \ell}$, which can be derived from the retarded Green function for transverse traceless (TT) metric perturbations via the Kubo formula

$$
\eta_{i j, k \ell}=-\lim _{\omega \rightarrow 0} \frac{1}{\omega} \operatorname{Im}\left[G_{i j, k \ell}(\omega, 0)\right] .
$$

The retarded Green function can in turn be obtained from the correlation function of two energy-momentum tensors for the transverse traceless modes, with

$$
G_{i j, k \ell}(\omega, 0)=\int d t d \vec{x} e^{i \omega t} \theta(t)\left\langle\left[T_{i j}(t, \vec{x}), T_{k \ell}(0,0)\right]\right\rangle .
$$

Through the holographic duality, the two-point function is derivable from the bulk quadratic action for the metric perturbations, which in momentum space takes the form

$$
S^{(2)}=-\frac{1}{2} \int \frac{d^{4} k}{(2 \pi)^{4}} h_{i j}(k) \mathcal{F}_{i j, k \ell}(k, r) h_{k \ell}(-k) .
$$

\footnotetext{
${ }^{1} \mathrm{~A}$ violation of the viscosity bound has also recently been observed in a Horndeski gravity theory, whose action is purely linear in curvature [26, 27].
} 
One then has

$$
G_{i j, k \ell}(\omega, \vec{k})=\lim _{r \rightarrow \infty} \mathcal{F}_{i j, k \ell}(k, r) .
$$

(Some references where these results are discussed, both for the isotropic and the anisotropic cases, include [9, 15, 20, 25].)

In the anisotropic background solutions we shall consider in this paper, many of the components of TT metric fluctuations decouple from one another, and the shear viscosity tensor $\eta_{i j, k \ell}$ correspondingly is mostly diagonal. In some cases we encounter modes that cross-couple in a pairwise fashion, and these are associated with off-diagonal as well as diagonal entries. We find that some of the TT modes give rise to $\eta_{i j, k \ell}$ components that violate the KSS bound, while others give the standard $1 /(4 \pi)$ result.

The paper is organized as follows. In section 2, we consider AdS black holes in $n=$ $N p+2$ dimensions carrying magnetic $p$-form charges. In section 3 , we study the linearised gravitational TT modes, and show that they obey a Klein-Gordon equation with a positiondependent mass. In section 4 , we calculate the viscosity/entropy ratio, and evaluate it in various limits and special cases. We conclude the paper in section 5 . New classes of Lifshitz black holes with hyperscaling behaviour in theories of gravity with form fields and a dilaton, where the form fields carrying magnetic charges, are given in an appendix.

\section{AdS planar black holes with magnetic charges}

In this section we consider Einstein gravity coupled to $N p$-form field strengths $F_{(p)}^{I}=$ $d A_{(p-1)}^{I}, I=1,2, \ldots, N$, with the Lagrangian

$$
\mathcal{L}=\sqrt{-g}\left(R-2 \Lambda-\frac{1}{2 p !} \sum_{I=1}^{N}\left(F_{(p)}^{I}\right)^{2}\right) .
$$

A general class of AdS black branes were constructed in [30]. For simplicity, we assume that the spacetime dimension is

$$
n=N p+2 .
$$

The theory admits AdS planar black hole solutions with the metric ansatz given by

$$
d s_{n}^{2}=-h d t^{2}+\frac{d r^{2}}{f}+r^{2} d \Sigma^{2}
$$

where $d \Sigma^{2}$ is the metric on an $(n-2)$-dimensional Euclidean space. The Einstein tensor $G_{\mu \nu}=R_{\mu \nu}-\frac{1}{2} R g_{\mu \nu}$ has non-zero components given by

$$
\begin{aligned}
G_{t t} & =-\frac{(n-2)}{2 r} h f^{\prime}-\frac{(n-2)(n-3)}{2 r^{2}} h f \\
G_{r r} & =\frac{(n-2)}{2 r} \frac{h^{\prime}}{h}+\frac{(n-2)(n-3)}{2 r^{2}}, \\
G_{i j} & =\left[\frac{(n-3) r}{2}\left(f^{\prime}+\frac{f h^{\prime}}{h}\right)+\frac{r^{2} f h^{\prime \prime}}{2 h}+\frac{r^{2} f^{\prime} h^{\prime}}{4 h}-\frac{r^{2} f h^{\prime 2}}{4 h^{2}}+\frac{1}{2}(n-3)(n-4) f\right] \delta_{i j} .
\end{aligned}
$$


Here, we shall consider the case where the $N p$-form field strengths each carry a magnetic charge and each spans a disjoint $p$-dimensional subspace of the $(N p)$-dimensional Euclidean space. Since the metric ansatz $(2.3)$ is isotropic in the $(n-2)$ transverse directions, the $N$ magnetic charges must necessarily be equal. Thus we may write

$$
\begin{aligned}
& d \Sigma^{2}=d \Sigma_{1}^{2}+d \Sigma_{2}^{2}+\cdots d \Sigma_{N}^{2} \\
& d \Sigma_{1}^{2}=d x_{1}^{2}+\cdots+d x_{p}^{2}, \quad d \Sigma_{2}^{2}=d y_{1}^{2}+\cdots+d y_{p}^{2}, \quad \text { etc. }
\end{aligned}
$$

with the magnetically charged field strengths given by

$$
\begin{aligned}
& F_{(p)}^{I}=\alpha \Sigma_{(p)}^{I}, \quad I=1,2, \ldots, N, \\
& \Sigma_{(p)}^{1}=d x_{1} \wedge \cdots \wedge d x_{p}, \quad \Sigma_{(p)}^{2}=d y_{1} \wedge \cdots \wedge d y_{p}, \quad \text { etc. }
\end{aligned}
$$

The black hole solution is then given by

$$
h=f=g^{2} r^{2}-\frac{\alpha^{2}}{\Delta r^{2 p-2}}-\frac{\mu}{r^{n-3}}, \quad \Delta=2 p(n-2 p-1)=2(N-2) p^{2}+2 p,
$$

where $\ell=1 / g$ is the AdS radius, defined by

$$
g^{2}=-\frac{2 \Lambda}{(n-1)(n-2)} .
$$

Note that the $p=1$ case corresponds to the linear axion models [2].

The $(n-2)$-dimensional metric $d \Sigma^{2}$ on the transverse space has the full isometries of the $(n-2)$-dimensional Euclidean group $\mathbb{E}^{n-2}$, namely, the semi-direct product of the translations $\mathbb{R}^{n-2}$ with the rotation group $\mathrm{SO}(n-2)=\mathrm{SO}(N p)$. The ansatz (2.6) for the $N p$-form field strengths, on the other hand, breaks the $\mathrm{SO}(N p)$ rotational symmetry of the complete solution down to $\operatorname{SO}(p)^{N}$. Since the gauge potentials $A_{(p-1)}^{I}$ enter the equations of motion only via their gauge-invariant field strengths $F_{(p)}^{I}=d A_{(p-1)}^{I}$, we may view the translational symmetries of the $(n-2)$-dimensional transverse space as remaining unbroken in the bulk solution, modulo gauge transformations. ${ }^{2}$ The case when $p=1$ is special; this corresponds to $N$ 1-form field strengths $F_{(1)}^{I}=d \chi^{I}$. The scalar Lagrangian has a global $\mathbb{E}^{N}$ symmetry, comprising the semi-direct product of the axionic shift symmetries $\mathbb{R}^{N}$ and the internal $\mathrm{SO}(N)$ rotations. Setting $\chi^{I}=\alpha x^{I}$ equates the $\mathbb{E}^{N}$ internal scalar symmetries with the $\mathbb{E}^{N}$ symmetries of the Euclidean transverse metric $d x^{I} d x^{I}$, leading to a spontaneous breaking of $\mathbb{E}^{N} \times \mathbb{E}^{N}$ to the diagonal $\mathbb{E}^{N}$ subgroup. As far as the bulk solution is concerned, one could choose to attribute this to a breaking of the internal scalar symmetries, with the Euclidean symmetries of the transverse space remaining unbroken. However, from the point of view of the CFT, the explicit coordinate dependence of the

\footnotetext{
${ }^{2}$ One could take, for example, $A_{(p-1)}^{1}=\alpha x_{1} d x_{2} \wedge \cdots \wedge d x_{p}$, which is not itself translationally invariant under $x_{1} \longrightarrow x_{1}+c_{1}$, but it is invariant modulo the gauge transformation $A_{(p-1)}^{1} \longrightarrow A_{(p-1)}^{1}+d \Lambda_{(p-2)}^{1}$, with $\Lambda_{(p-2)}^{1}=-c_{1} x_{2} d x_{3} \wedge \cdots \wedge d x_{p}$. Note that although the bulk solutions can be viewed as being translationally invariant, translational symmetry is broken in the boundary CFTs, since the potentials themselves are the sources dual to operators in the boundary theory. A review of theories with broken translation invariance can be found in [31].
} 
scalars $\chi^{I}$ implies that the dual operators in the boundary theory break the translational symmetries.

For $p \geq 2$, the metric (2.3) with (2.7) can instead be supported by a lesser number of field strengths. For example, we can use just one $p$-form field strength, with

$$
F_{(p)}^{1}=\alpha\left(\Sigma_{(p)}^{1}+\Sigma_{(p)}^{2}+\cdots+\Sigma_{(p)}^{N}\right) .
$$

The $p=2$ solution with a single Maxwell field was constructed in [1]. The general $p$-form solutions were previously constructed in [30]. (See also [32, 33].) As we shall see later, although the black hole metric is the same for these different form-field configurations, a transverse traceless metric perturbation can, under certain circumstances, be different, leading to a non-isotropic shear viscosity tensor. For now, we note that for $p \geq 3$ the expression (2.9) is invariant under the $\mathrm{SO}(p)^{N}$ subgroup of the $\mathrm{SO}(N p)$ rotational symmetry group of the transverse Euclidean space. The case $p=2$ is special, as we shall discuss later, with the symmetry now becoming $\mathrm{U}(N)$.

It is worth pointing out that in our construction or in that of [1], the spacetime dimensions are forced to be $D=N p+2$ for given $p$-forms. In particular this rules out $D=5$ for $p=2$. However, isotropic AdS black branes with magnetic charges can be constructed [34] in the $\mathrm{U}(1)^{3}$ gauged supergravity that can be obtained from the $S^{5}$ reduction of type IIB supergravity [35].

\section{Transverse, traceless perturbations}

As discussed in the introduction, the components of the shear viscosity tensor $\eta_{i j, k \ell}$ can be calculated by means of the holographic prescription, by obtaining the quadratic terms in the action that describes the transverse traceless metric fluctuations around the backgrounds we have constructed in section 2. To obtain these modes, we replace the metric $(2.3)$ by $^{3}$

$$
d s^{2}=-h d t^{2}+\frac{d r^{2}}{f}+r^{2}\left(d \Sigma_{n-2}^{2}+2 \Psi(r, t) d x d y\right) .
$$

Here, $x$ and $y$ represent, for now, any two out of the total set of $(n-2)$ coordinates in the Euclidean space of the $d \Sigma_{n-2}^{2}$ metric. The original Ricci tensor of the background metric acquires a single non-vanishing correction at linear order in $\Psi$, namely

$$
\begin{aligned}
R_{x y}^{(1)} & =-\frac{1}{2} r^{2} \square \Psi-\frac{1}{2} r f^{\prime} \Psi-\frac{r f h^{\prime}}{2 h} \Psi-(n-2) f \Psi \\
& =-\frac{1}{2} r^{2} \square \Psi+R_{x x}^{(0)} \Psi
\end{aligned}
$$

where $R_{x x}^{(0)}$ denotes any of the (diagonal) components of the zeroth-order Ricci tensor of the background metric, in the directions of the transverse $(n-2)$-dimensional Euclidean

\footnotetext{
${ }^{3}$ For now, we consider a perturbation $\delta g_{x y}$ involving just a single component of the metric. Later, we shall encounter a case where it is necessary to consider perturbations with two non-zero components, $\delta g_{x_{1} y_{1}}$ and $\delta g_{x_{2} y_{2}}$.
} 
space. Thus the Einstein equation $G_{\mu \nu}=\frac{1}{2} T_{\mu \nu}$, which can be graded in orders of powers of $\Psi$ to give $G_{\mu \nu}^{(0)}=\frac{1}{2} T_{\mu \nu}^{(0)}, G_{\mu \nu}^{(1)}=\frac{1}{2} T_{\mu \nu}^{(1)}$ and so on, leads to

$$
\begin{aligned}
G_{x y}^{(1)} & =-\frac{1}{2} r^{2} \square \Psi+R_{x x}^{(0)} \Psi-\frac{1}{2} R^{(0)} g_{x y}^{(1)}, \\
& =-\frac{1}{2} r^{2} \square \Psi+G_{x x}^{(0)} \Psi \\
& =-\frac{1}{2} r^{2} \square \Psi+\frac{1}{2} T_{x x}^{(0)} \Psi
\end{aligned}
$$

and hence

$$
\square \Psi-\frac{1}{r^{2}}\left(T_{x x}^{(0)} \Psi-T_{x y}^{(1)}\right)=0 .
$$

Writing $T_{x y}^{(1)}=\left(\delta T_{x y} / \delta g_{x y}\right) \delta g_{x y}$ gives, as in [3],

$$
\square \Psi-m^{2}(r) \Psi=0,
$$

where

$$
m(r)^{2}=g^{x x} T_{x x}-\frac{\delta T_{x y}}{\delta g_{x y}},
$$

which reproduces the result in [3]. In the metric background (2.3), eqn (3.5) is given by

$$
f \Psi^{\prime \prime}+\left(\frac{f h^{\prime}}{2 h}+\frac{1}{2} f^{\prime}+\frac{(n-2)}{r} f\right) \Psi^{\prime}-\frac{1}{h} \ddot{\Psi}-m(r)^{2} \Psi=0,
$$

where a dot denotes a derivative with respect to $t$.

The energy-momentum tensor for the set of $N p$-form field strengths is given by

$$
T_{\mu \nu}=\frac{1}{(p-1) !} \sum_{I=1}^{N}\left[F_{\mu \rho_{2} \cdots \rho_{p}}^{I} F_{\nu}^{I \rho_{2} \cdots \rho_{p}}-\frac{1}{2 p}\left(F^{I}\right)^{2} g_{\mu \nu}\right] \text {. }
$$

With the fields given by (2.6), we therefore have that in the background solution,

$$
T_{x_{1} x_{1}}=T_{x_{2} x_{2}}=\cdots=T_{y_{1} y_{1}}=T_{y_{2} y_{2}}=\cdots=\frac{\alpha^{2}\left(1-\frac{1}{2} N\right)}{r^{2 p-2}} .
$$

We may now consider various different classes of transverse traceless normal modes, which will give different expressions for the corresponding components of the shear viscosity tensor $\eta_{i j, k \ell}$ :

Class 1: if we consider a TT metric fluctuation such as $\delta g_{x_{1} y_{1}}$ (or any other case where one index is chosen from the range of coordinates spanned by one of the field strengths, and the other index chosen from the range of coordinates spanned by another field strength), then the first-order fluctuation in $T_{\mu \nu}$ will come purely from the fluctuation of $g_{\mu \nu}$ in the $F^{2} g_{\mu \nu}$ terms in (3.8), and hence we shall have

$$
T_{x_{1} y_{1}}=-\frac{\alpha^{2} N}{2 r^{2 p}} \delta g_{x_{1} y_{1}} .
$$


Thus, substituting into the expression (3.6), we find

$$
m(r)^{2}=\frac{\alpha^{2}}{r^{2 p}}
$$

The corresponding calculation of the shear viscosity associated with this mode, using the Kubo formula discussed in the Introduction, will therefore provide an expression for the component $\eta_{x_{1} y_{1}, x_{1} y_{1}}$ of the shear viscosity tensor $\eta_{i j, k \ell}$. This is the generalisation, to the case of $N p$-form field strengths, of the situation with $N$ axionic scalars, which can be viewed as the 0 -form potentials for 1-form field strengths, which was considered in [3]. We shall discuss the explicit calculation of the shear viscosity component in section 4 .

Class 2: an alternative possibility for the TT perturbation, keeping the same configuration (2.6) for the field strengths, is to take both of the indices on the metric fluctuation to lie in a range spanned by a single field strength. (This requires that $p \geq 2$.) Let us consider, for example, $\delta g_{x_{1} x_{2}}$. The first-order fluctuation of the energy-momentum tensor will now receive contributions from both the terms inside the summation in (3.8), giving

$$
T_{x_{1} x_{2}}=\frac{\alpha^{2}\left(1-\frac{1}{2} N\right)}{r^{2 p}} \delta g_{x_{1} x_{2}} .
$$

Substituting this result, together with (3.9), into (3.6) then gives

$$
m(r)^{2}=0
$$

for this case. The result for the corresponding shear viscosity component $\eta_{x_{1} x_{2}, x_{1} x_{2}}$ will be discussed in section 4 .

One can also consider other possible form-field configurations that will give rise to black hole solutions with the metric taking the spatially-isotropic form (2.3), such as the case of a single $p$-form field strength spanning an $(N p)$-dimensional transverse space, as in (2.9). It is easy to verify that if $p \geq 3$, this gives the same results for $m(r)^{2}$ as we obtained in the previous example with $N$ distinct $p$-forms each spanning non-overlapping $p$-dimensional subspaces. Namely, with the ansatz (2.9) for a single $p$-form, we find:

Class 3: if the two indices on the metric perturbation are taken from two different $p$ dimensional subspaces, giving rise to a shear viscosity component $\eta_{x_{1} y_{1}, x_{1} y_{1}}$, then $m(r)^{2}$ is non-zero and given by (3.11),

and

Class 4: if the two indices on the metric perturbation lie within the same subspace, giving rise to a shear viscosity component $\eta_{x_{1} x_{2}, x_{1} x_{2}}$, then $m(r)^{2}$ vanishes as in (3.13).

We can also have the situation in: 
Class 5: a special case arises if a 2-form field strength spans a $2 N$-dimensional transverse space, as in (2.9). Now, unlike the situation when $p \geq 3$, the term $F_{\mu \rho_{2} \cdots \rho_{p}} F_{\nu}{ }^{\rho_{2} \cdots \rho_{p}}$ in the energy-momentum tensor will also give a contribution at linear order in a metric perturbation $\delta g_{x y}$ where the two indices lie in different 2-dimensional subspaces. Thus if the transverse directions are $\left(x_{1}, x_{2}, y_{1}, y_{2}, \cdots\right)$, and we consider a metric fluctuation $\delta g_{x_{1} y_{1}}$, then there will be linear-order terms in the energy momentum tensor given by

$$
T_{x_{1} y_{1}}^{(1)}=-\frac{N \alpha^{2}}{2 r^{4}} \delta g_{x_{1} y_{1}}, \quad T_{x_{2} y_{2}}^{(1)}=-\frac{\alpha^{2}}{r^{4}} \delta g_{x_{1} y_{1}} .
$$

In order to obtain TT eigenfunctions of the linearised fluctuation equations, it is therefore necessary to consider metric fluctuations with both $\delta g_{x_{1} y_{1}}$ and $\delta g_{x_{2} y_{2}}$ nonzero in this case. The corresponding TT Lichnerowicz modes are diagonalised by writing $\delta g_{x_{1} y_{1}}=\frac{1}{2} r^{2}\left(\Psi_{+}+\Psi_{-}\right)$and $\delta g_{x_{2} y_{2}}=\frac{1}{2} r^{2}\left(\Psi_{+}-\Psi_{-}\right)$, for which, using also the appropriate extension of (3.4), we find

$$
\square \Psi_{ \pm}-m_{ \pm}(r)^{2} \Psi_{ \pm}=0
$$

with

$$
m_{+}(r)^{2}=\frac{2 \alpha^{2}}{r^{4}}, \quad m_{-}(r)^{2}=0 .
$$

Note that in this case the fact that the TT mode involves excitations both of $\delta g_{x_{1} y_{1}}$ and $\delta g_{x_{2} y_{2}}$ means that there will be off-diagonal as well as diagonal terms in the quadratic action (1.4), and hence also in the associated components of $\eta_{i j, k \ell}$, namely

$$
\left(\begin{array}{ll}
\eta_{x_{1} y_{1}, x_{1} y_{1}} & \eta_{x_{1} y_{1}, x_{2} y_{2}} \\
\eta_{x_{2} y_{2}, x_{1} y_{1}} & \eta_{x_{2} y_{2}, x_{2} y_{2}}
\end{array}\right) .
$$

It is worth remarking that the single 2 -form field with the ansatz (2.9) in the $2 N$ dimensional transverse space also breaks the $\mathrm{SO}(2 N)$ rotational symmetry of the background metric. In this case, it is broken down to $\mathrm{U}(N)$, as may be seen by introducing complex coordinates $z_{a}$ defined by

$$
z_{1}=x_{1}+\mathrm{i} x_{2}, \quad z_{2}=y_{1}+\mathrm{i} y_{2}, \quad \text { etc. },
$$

in terms of which the field strength can be written as

$$
F_{(2)}=\frac{\mathrm{i} \alpha}{2} d z_{a} \wedge d \bar{z}_{a} .
$$

Thus $F_{(2)}$ can be seen to be $\alpha$ times the Kähler form on the $2 N$-dimensional Euclidean space, and it manifestly has a $\mathrm{U}(N)$ symmetry.

\section{Viscosity/entropy ratio}

In the previous sections, we consdered magnetically charged black branes and analyzed the transverse and traceless perturbation modes. Now, we turn to the derivation of the viscosity to entropy density ratio. Usually, it is a universal value (1.1) for gauge theories which have two-derivative Einstein gravity dual. However, as is observed in [3], and as we discussed earlier, the situation is changed when the theory involves anisotropic matter fields. 


\subsection{A general discussion}

As discussed in [3], and the previous section, a traceless and transverse mode in the metric (2.3) satisfies (3.5). Consider $\Psi(r, t)=e^{-\mathrm{i} \omega t} \psi(r)$. Near the horizon with

$$
h=h_{1}\left(r-r_{0}\right)+h_{2}\left(r-r_{0}\right)^{2}+\cdots, \quad f=f_{1}\left(r-r_{0}\right)+f_{2}\left(r-r_{0}\right)^{2}+\cdots,
$$

we have

$$
\psi(r) \sim \exp \left(-\frac{\mathrm{i} \omega}{4 \pi T} \log \left(r-r_{0}\right)\right)
$$

where

$$
T=\frac{\sqrt{f_{1} h_{1}}}{4 \pi}
$$

is the Hawking temperature. This leads to the ansatz for $\psi$ in a small- $\omega$ expansion, with

$$
\psi(r)=\chi(r) \exp \left(-\frac{\mathrm{i} \omega}{4 \pi T} \log f(r)\right)(1-\mathrm{i} \omega U(r))+\mathcal{O}\left(\omega^{2}\right),
$$

where $\chi$ is the solution of equation (3.7) with $\omega=0$ :

$$
f \chi^{\prime \prime}+\left(\frac{f h^{\prime}}{2 h}+\frac{1}{2} f^{\prime}+\frac{(n-2)}{r} f\right) \chi^{\prime}-m(r)^{2} \chi=0 .
$$

For the massless case with $m(r)=0, \chi$ is simply a constant, and can be set to be 1 . For non-vanishing $m(r), \chi$ depends on $r$. If $m(r)$ falls off sufficiently fast asymptotically, then $\chi \rightarrow 1$ as $r \rightarrow \infty$. The function $U$ can also be solved up to quadratures, and is given by

$$
U^{\prime}=\frac{1}{r^{n-2} \chi^{2} \sqrt{h f}}\left(c-\frac{\chi^{2} f^{\prime} r^{n-2}}{4 \pi T} \sqrt{\frac{h}{f}}\right),
$$

where the integration constant $c$ should be chosen such that the zero in the denominator coming from the vanishing of $f$ and $h$ on the horizon is cancelled. Thus we have

$$
c=r_{0}^{n-2} \chi\left(r_{0}\right)^{2}=4 S \chi\left(r_{0}\right)^{2} .
$$

where $S$ is the entropy density, given by

$$
S=\frac{1}{4} r_{0}^{n-2}
$$

This implies

$$
\psi^{*} \psi^{\prime}=-\frac{4 \mathrm{i} \omega S \chi\left(r_{0}\right)^{2}}{\sqrt{h f} r^{n-2}}+\chi \chi^{\prime}+\mathcal{O}\left(\omega^{2}\right) .
$$

If we now implement the procedure outlined in the Introduction, based on the use of the Kubo formula as described in [9, 15], then for the black holes with the metric ansatz (2.3) in [12], the shear viscosity component $\eta$ corresponding to a particular transverse traceless normal mode will be given by

$$
\eta=-\lim _{r \rightarrow \infty}\left[\frac{r^{n-2} \sqrt{h f}\left(\psi^{*} \psi^{\prime}-\text { c.c. }\right)}{32 \mathrm{i} \pi \omega}\right]=\frac{S}{4 \pi} \chi\left(r_{0}\right)^{2},
$$


and hence

$$
\frac{\eta}{S}=\frac{\chi\left(r_{0}\right)^{2}}{4 \pi}
$$

We now apply the above discussion to the AdS planar black holes in section 2. The metric functions were given in (2.7), and $m(r)$ is given by

$$
m(r)^{2}=\frac{\nu \alpha^{2}}{r^{2 p}}
$$

The parameter $\nu$ can be 0,1 or 2 , corresponding to the cases we saw in (3.13), (3.11) and the $m_{+}(r)^{2}$ expression in (3.16) respectively. (The $\nu=2$ case arises only when $p=2$.) As can be seen from (4.5), the value of $\chi\left(r_{0}\right)$, which determines the result for the viscosity component $\eta$ in (4.10), is completely governed by the value of $\nu$ corresponding to the normal mode under discussion, once the metric functions $f$ and $h$ in the background solution (2.7) are known. Thus for the five classes of TT metric perturbations discussed in section 3, we shall have

$$
\begin{aligned}
\text { Class 1 \& Class 3: } & \eta_{x_{1} y_{1}, x_{1} y_{1}}=\left.\eta\right|_{\nu=1}, \\
\text { Class 2 \& Class 4: } & \eta_{x_{1} x_{2}, x_{1} x_{2}}=\left.\eta\right|_{\nu=0}, \\
\text { Class 5: } & \eta_{x_{1} y_{1}, x_{1} y_{1}}=\eta_{x_{2} y_{2}, x_{2} y_{2}}=\frac{1}{2}\left(\eta_{+}+\eta_{-}\right), \\
\eta_{x_{1} y_{1}, x_{2}, y_{2}} & =\eta_{x_{2} y_{2}, x_{1} y_{1}}=\frac{1}{2}\left(\eta_{-}-\eta_{+}\right),
\end{aligned}
$$

where in the case of Class 5 we have

$$
\eta_{+}=\left.\eta\right|_{\nu=2}, \quad \eta_{-}=\left.\eta\right|_{\nu=0} .
$$

Our results for $\eta$, as functions of the quantity $\nu$, are given in the following three subsections. The implications for the various components of the viscosity tensor $\eta_{i j, k \ell}$ can then be read off from (4.13).

\subsection{The special case $\mu=0$}

We first consider the case where $\mu=0$ in the metric functions given in (2.7). The horizon is located at $r_{0}$ with

$$
r_{0}^{2 p}=\frac{\alpha^{2}}{\Delta g^{2}},
$$

and the Hawking temperature is

$$
T=\frac{p g^{2}}{2 \pi \Delta^{\frac{1}{2 p}}}\left(\frac{\alpha}{g}\right)^{\frac{1}{p}} .
$$

The function $\chi$ can be found explicitly in this case, and is given by

$$
\begin{aligned}
\chi= & c_{12} F_{1}\left[\frac{1}{2}(1-x-y), \frac{1}{2}(1-x+y) ; 1-x ;\left(\frac{r_{0}}{r}\right)^{2 p}\right] \\
& +\frac{c_{2}}{r^{2 p x}}{ }_{2} F_{1}\left[\frac{1}{2}(1+x-y), \frac{1}{2}(1+x+y) ; 1+x ;\left(\frac{r_{0}}{r}\right)^{2 p}\right],
\end{aligned}
$$


where $c_{1}$ and $c_{2}$ are two integration constants and

$$
x=\frac{n-1}{2 p}, \quad y=\frac{\sqrt{(n-2 p-1)(n-2(4 \nu+1) p-1)}}{2 p} .
$$

The quantity $\chi$ diverges when $r=r_{0}$ for general values of $c_{1}$ and $c_{2}$, but it can be made convergent by choosing

$$
\frac{c_{2}}{c_{1}}=-\frac{\Gamma(1-x) \Gamma\left(\frac{1}{2}(1+x-y)\right) \Gamma\left(\frac{1}{2}(1+x+y)\right)}{\Gamma(1+x) \Gamma\left(\frac{1}{2}(1-x-y)\right) \Gamma\left(\frac{1}{2}(1-x+y)\right)} \frac{1}{r_{0}^{2 p x}} .
$$

With this choice, we find

$$
\chi\left(r_{0}\right)=\frac{2 c_{1} \pi \Gamma(1-x) \sin (\pi x)}{(\cos (\pi x)+\cos (\pi y)) \Gamma\left(\frac{1}{2}(1-x-y)\right) \Gamma\left(\frac{1}{2}(1-x+y)\right.} .
$$

Thus for this case, we have

$$
\frac{4 \pi \eta}{S}=\left(\frac{2 \pi \Gamma(1-x) \sin (\pi x)}{(\cos (\pi x)+\cos (\pi y)) \Gamma\left(\frac{1}{2}(1-x-y)\right) \Gamma\left(\frac{1}{2}(1-x+y)\right)}\right)^{2},
$$

which is less than 1 if $\nu>0$. The result with $p=1$ in four dimensions was obtained previously in [36]. Thus the viscosity tensor components in the classes 1 and 3 that we defined in section 3 , together with the diagonal components in class 5 , violate the viscosity/entropy bound.

Turning now to the case where the parameter $\mu$ in the metric functions $h$ and $f$ in (2.7) is non-zero, it is no longer possible to obtain a general closed-form expression for $\chi(r)$. It is nevertheless possible to obtain expansions valid for small values of the magnetic charge parameter $\alpha$, or else for small values of the Hawking temperature of the black hole, as we shall discuss below.

\subsection{Small $\alpha$ (large $T$ ) expansion}

The metric functions (2.7), and the other quantities needed for solving (4.5), are given by

$$
h=f=g^{2} r^{2}-\frac{\alpha^{2}}{\Delta r^{2 p-2}}-\frac{\mu}{r^{n-3}}, \quad m(r)^{2}=\frac{\nu \alpha^{2}}{r^{2 p}}, \quad \Delta=2 p(n-2 p-1) .
$$

The horizon is located at $r=r_{0}$, implying that $\mu$ is given by

$$
\mu=g^{2} r_{0}^{n-1}\left(1-\frac{\alpha^{2}}{\Delta g^{2} r_{0}^{2 p}}\right),
$$

and hence the Hawking temperature $T=h^{\prime}\left(r_{0}\right) /(4 \pi)$ is given by

$$
T=\frac{(n-1) g^{2} r_{0}}{4 \pi}\left[1-\frac{\alpha^{2}}{2 p(n-1) g^{2} r_{0}^{2 p}}\right] \text {. }
$$

Defining $T_{0}$ to be the temperature when $\alpha=0$, we have

$$
T=T_{0}\left[1-\frac{1}{8 \pi p}\left(\frac{(n-1) g^{2}}{4 \pi}\right)^{2 p-1}\left(\frac{\alpha}{T_{0}^{p}}\right)^{2}\right], \quad T_{0}=\frac{(n-1) g^{2} r_{0}}{4 \pi} .
$$

If $\alpha / T_{0}^{p} \ll 1$ then we have $T \approx T_{0}$, with $T \leq T_{0}$, and so if $\alpha / T^{p} \ll 1$ we also have $\alpha / T_{0}^{p} \ll 1$. 
We may seek a solution for the function $\chi$ as a series expansion in powers of $\alpha$, writing

$$
\chi=1+\alpha^{2} \tilde{\chi}+\cdots .
$$

Substituting into (4.5), we find that $\tilde{\chi}$ has the solution

$$
\tilde{\chi}(z)=-\frac{\nu}{g^{2}(n-1-2 p) r_{0}^{2 p}}\left[\left(\frac{z^{2 p}}{2 p}\right){ }_{2} F_{1}\left[1, \frac{2 p}{n-1} ; 1+\frac{2 p}{n-1} ; z^{n-1}\right]+\frac{1}{n-1} \log \left(1-z^{n-1}\right)\right],
$$

where $z=r_{0} / r$. Thus on the horizon, at $z=1$, we have

$$
\tilde{\chi}(1)=\frac{\nu\left[\gamma+P\left(\frac{2 p}{n-1}\right)\right]}{(n-1)(n-2 p-1) g^{2} r_{0}^{2 p}},
$$

where $\gamma$ is the Euler-Mascheroni constant and $P(z) \equiv \Gamma^{\prime}(z) / \Gamma(z)$ is the digamma function. This implies that the viscosity/entropy ratio is given by

$$
\frac{4 \pi \eta}{S}=1+\frac{2 \nu(n-1)^{2 p-1} g^{4 p-2}}{(n-2 p-1)(4 \pi)^{2 p}}\left[\gamma+P\left(\frac{2 p}{n-1}\right)\right]\left(\frac{\alpha}{T^{p}}\right)^{2}+\mathcal{O}\left(\left(\frac{\alpha}{T^{p}}\right)^{4}\right) .
$$

Note that the value of $\gamma+P\left(\frac{2 p}{n-1}\right)$ is always less than zero (for the relevant values of $n$ and $p$ ), so the ratio $4 \pi \eta / S$ is less than 1 when $\nu>0$. This implies that the viscosity tensor components in the classes 1 and 3, and the diagonal components in class 5, which we defined in section 3, all violate the viscosity/entropy bound. The result for the special $n=4$ and $p=1$ case was obtained in [3].

\subsection{Low temperature expansion}

When the magnetic charge parameter $\alpha$ is given by

$$
\alpha^{2}=2(n-1) p g^{2} r_{0}^{2 p}
$$

the solution becomes extremal with zero temperature. In this case, viscosity vanishes. At the low-temperature, we find that the equation for $\chi$ seemingly cannot be solved analytically, except when $p=1$, with $n=4$ or 5 . The four-dimensional result was given in [3]. In five dimensions (with $\nu=1$ ), we find

$$
\chi(z)=\frac{1-3 z^{2}}{1-z^{2}}-\frac{4 z^{4} \log z}{\left(1-z^{2}\right)^{2}}+\frac{2 \pi T z^{2}\left(1-z^{4}+4 z^{2} \log z\right)}{g^{2} r_{0}\left(1-z^{2}\right)^{3}}+\mathcal{O}\left(T^{2}\right),
$$

where again, $z=r_{0} / r$. This means that at on the horizon (i.e. at $z=1$ ),

$$
\chi(1)=-\frac{2 \pi T}{3 g^{2} r_{0}},
$$

and hence, from (4.11), we find

$$
\frac{4 \pi \eta}{S}=\frac{4 \pi^{2} T^{2}}{9 g^{4} r_{0}^{2}}+\cdots=\frac{32 \pi^{2}}{9 g^{2}}\left(\frac{T}{\alpha}\right)^{2}+\mathcal{O}\left(\left(\frac{T}{\alpha}\right)^{4}\right) .
$$


Thus the viscosity bound (1.1) is violated in the special case when $\mu=0$, and in the high-temperature limit, and also in the low-temperature limit where the viscosity/entropy ratio is proportional to $T^{2}$. The low-temperature result provides further examples of the phenomenon seen already in [3] and elsewhere, that the ratio $\eta / S$ can not only be less than the KSS bound $1 /(4 \pi)$, but it can be arbitrarily close to zero. ${ }^{4}$

\section{Conclusions}

In this paper, we studied $n$-dimensional magnetically-charged planar black hole solutions in the theory of Einstein gravity coupled to $N p$-form field strengths. In general, turning on the magnetic charges would result in solutions where the metric in the transverse space would be anisotropic. However, by requiring the field strengths to span the entire transverse space, with equal magnetic charges associated with each of the $p$-dimensional planes, we obtained solutions where the metric was isotropic in the transverse dimensions, with the full Euclidean $\mathbb{E}^{n-2}$ symmetry. The field strengths in the solution in general break the associated $\mathrm{SO}(n-2)$ rotational symmetry of the metric to a subgroup.

We then studied the transverse and traceless metric perturbations of the background solutions. Although the metric is isotropic, the linearised TT modes turn out to be satisfy scalar wave equations with different radially-dependent "mass terms" that are proportional to the magnetic charge parameter $\alpha$ as in (4.12). For $p=1$ forms, corresponding to axion potentials, we have $\nu=1$; for $p=2$ Maxwell field strengths, we have $\nu=0,1$ or 2, depending on the nature of the charge configurations; for $p \geq 3$, we have $\nu=0$ or 1 . The shear viscosity tensor can be calculated by means of the Kubo formula, and its components turn out to depend on the magnitudes of the zero-frequency linearised TT modes evaluated on the horizon. It can be easily established that the $m(r)=0$ cases yield components with the standard KSS ratio $\eta / S=1 /(4 \pi)$. For $m(r)^{2}>0$, the general linearised equation cannot be solved analytically, although there is a special case where the mass parameter $\mu$ in the black hole solution vanishes, for which we can obtain an analytical solution for the linearised mode. We then studied the cases where $\mu \neq 0$, for which we could solve the linearised equation in the limits of a high-temperature expansion and also in a lowtemperature expansion. In both cases, the viscosity bound is violated, and the ratio $\eta / S$ approaches zero, proportional to $T^{2}$, in the low-temperature limit.

It is worth commenting that the underlying reason for the violation of the KSS bound is very different from the standpoint of the boundary field theory versus the bulk gravity. For example, for the linear axion models corresponding to $p=1$, the violation of the bound is attributed in the boundary field theory to the linear dependence of the axions on the spatial coordinates, which breaks the translational invariance. On the other hand, from the viewpoint of the bulk theory, one may view the translational symmetry as remaining unbroken, at the expense of the breaking of the axionic shift symmetries of the scalar fields. (Analogous statements are true also for all $p \geq 1$.) Furthermore, from the bulk viewpoint, the full $\mathrm{SO}(n-2)=\mathrm{SO}(N p)$ rotational symmetry in the transverse space may be considered to be preserved for $p=1$. For $p \geq 3$, the rotational symmetry breaks down

\footnotetext{
${ }^{4} \mathrm{~A}$ large violation of the KSS bound in a system without pathologies was first exhibited in [37].
} 
to $\mathrm{SO}(p)^{N}$. In this case, $m(r)^{2}$ for TT modes within a $p$-dimensional subspace spanned by a magnetic charge vanishes, while $m(r)^{2}$ is non-vanishing if the TT mode encompasses two distinct subspaces. The solution with a single $p=2$ field strength is more complicated since the rotational symmetry breaks down to $\mathrm{U}(N)$ in this case. In all cases, the violation of the KSS bound is related to the non-vanishing of $m(r)^{2}$; however, the direct culprit for such a non-vanishing mass in the bulk wave equation for the TT modes is not entirely clear. It is also worth commenting that the magnetically charged solutions break the scaling symmetry associated with the generalized Smarr relation that is the bulk dual to the KSS bound in a two-derivative gravity theory $[12,13]$.

We also derived magnetically-charged Lifshitz black holes with hyperscaling violation, which we presented in an appendix.

Since the matter fields are spatially dependent, the solutions we considered provide simple holographic models for studying momentum relaxation. It would also be interesting to study the transport properties of these solutions.

\section{Acknowledgments}

We are grateful to Sera Cremonini for helpful discussions. H-S.L. is supported in part by NSFC grants No. 11305140, 11375153, 11475148, 11675144 and CSC scholarship No. 201408330017. C.N.P. is supported in part by DOE grant DE-FG02-13ER42020. The work of H.L. is supported in part by NSFC grants NO. 11475024 and NO. 11235003.

\section{A Lifshitz black holes with hyperscaling violation}

In this section, we shall construct black hole solutions of the type (2.3), but carrying magnetic charges for form fields of different ranks. The energy-momentum tensors of the field strengths with different ranks have different fall-off rates, and it is necessary to introduce a dilaton field that can act as a compensator so that the metric in the $(n-2)$ brane directions can still be isotropic. Thus we shall consider the Lagrangian

$$
\mathcal{L}=\sqrt{-g}\left(R-2 \Lambda e^{a \phi}-\frac{1}{2}(\partial \phi)^{2}-\frac{1}{2} \sum_{i=1}^{N} \frac{1}{p_{i} !} e^{b_{i} \phi}\left(F_{\left(p_{i}\right)}^{i}\right)^{2}\right),
$$

where $a$ and $b_{i}$ are dilaton coupling constants. We assume that the spacetime dimension is

$$
n=2+\sum_{i=1}^{N} p_{i}
$$

A subset of the theories of the form (A.1) comprises those that are obtained from a circle reduction of the theories (2.1) that we studied earlier. For example, if we start from the $n$-dimensional theory of a single $p$-form coupled to gravity, then the theory in $n-1$ dimensions comprises a $p$-form, a $(p-1)$-form and a dilaton coupled to gravity. The resulting $(n-1)$-dimensional magnetic brane solution is also an asymptotically-AdS planar black hole, with $n=N p+2$. In this appendix, we shall not give the details of these 
solutions, since the dimensional reduction is straightforward. Instead we consider the more general Lagrangians that cannot be obtained from such a dimensional reduction.

We construct static and isotropic black holes with the metric form (2.3), with the ansatz for the metric and form fields being given by

$$
\begin{aligned}
d \Sigma^{2} & =\sum_{i=1}^{N} d \Sigma_{i}^{2}, \\
d \Sigma_{1}^{2} & =d x_{1}^{2}+\cdots+d x_{p_{1}}^{2}, \quad d \Sigma_{2}^{2}=d y_{1}^{2}+\cdots d y_{p_{2}}^{2}, \quad \text { etc. }, \\
F_{\left(p_{i}\right)}^{i} & =\alpha_{i} \Sigma_{i}, \\
\Sigma_{1} & =d x_{1} \wedge d x_{2} \wedge \cdots \wedge d x_{p_{1}}, \quad d \Sigma_{2}=d y_{1} \wedge d y_{2} \wedge \cdots \wedge d y_{p_{2}}, \quad \text { etc. }
\end{aligned}
$$

The equations of motion for the form fields are automatically satisfied. The scalar equation is given by

$$
\square \phi=2 a \Lambda e^{a \phi}+\sum_{i=1}^{N} \frac{b_{i} \alpha_{i}^{2} e^{b_{i} \phi}}{2 r^{2 p_{i}}} .
$$

The Einstein equations of motion are

$$
\begin{aligned}
G_{r r}-\phi^{\prime 2}-\frac{1}{2 f}\left(-2 \Lambda e^{a \phi}-\sum_{i=1}^{N} \frac{\alpha_{i}^{2} e^{b_{i} \phi}}{2 r^{2 p_{i}}}\right) & =0, \\
G_{t t}+\frac{1}{2} h\left(-2 \Lambda e^{a \phi}-\frac{1}{2} \phi^{\prime 2}-\sum_{i=1}^{N} \frac{\alpha_{i}^{2} e^{b_{i} \phi}}{2 r^{2 p_{i}}}\right) & =0, \\
G_{x_{i} x_{i}}-\frac{\alpha_{i}^{2} e^{b_{i} \phi}}{2 r^{2 p_{i}-2}}-\frac{1}{2} r^{2}\left(-2 \Lambda e^{a \phi}-\frac{1}{2} \phi^{\prime 2}-\sum_{i=1}^{N} \frac{\alpha_{i}^{2} e^{b_{i} \phi}}{2 r^{2 p_{i}}}\right) & =0,
\end{aligned}
$$

where $G_{\mu \nu}=R_{\mu \nu}-\frac{1}{2} R g_{\mu \nu}$ is the Einstein tensor. Since $G_{x_{i} x_{i}}$ in the different blocks $d \Sigma_{i}^{2}$ must the same for the isotropic solutions, the quantities

$$
\frac{\alpha_{i}^{2} e^{b_{i} \phi}}{r^{2 p_{i}-2}}
$$

must be the same for all $i=1,2, \ldots, N$. For general parameters, this implies that $\phi=$ $\gamma \log r$ with

$$
\alpha_{i}=\alpha, \quad \gamma b_{i}-2 p_{i}=\text { const. } \quad \text { for } \quad i=1,2, \ldots N .
$$

The constant $\gamma$ can be determined from the scalar equation, which implies

$$
\gamma b_{i}-2 p_{i}=\gamma a
$$

Summing over the all the $i$ indices yields

$$
\gamma=\frac{2(n-2)}{b-N a}, \quad b=\sum_{i=1}^{n} b_{i} .
$$


The magnetic charge parameter $\alpha$ is also fully determined, and is given by

$$
\alpha^{2}=\frac{4(-\Lambda)\left(N a^{2}-a b-2\right)}{N a b-2 N-b^{2}} .
$$

The metric functions $h$ and $f$ are given by

$$
h=r^{\frac{\gamma^{2}}{n-2}} f, \quad f=\sigma\left(r^{2+\gamma a}-\frac{\mu}{r^{n-3+\frac{\gamma^{2}}{2(n-2)}}}\right),
$$

where

$$
\sigma=\frac{8(n-2)^{2}(-\Lambda) a}{\gamma((n-2)(a b-2)+\gamma b)\left(2(n-1)(n-2)+2(n-2) a \gamma+\gamma^{2}\right)} .
$$

Thus the solutions are asymptotic to Lifshitz spacetimes [38, 39], with hyperscaling violation [40-43]. Such black hole solutions in the literature typically involve Maxwell fields carrying electric charges, whilst our solutions involve various $p$-forms that carry magnetic charges. Note that if $p_{i}=p$ for all $i$ (with either a single or else multiple $p$-form field strengths), we must have $\alpha_{i}=\alpha$ and $b_{i}=b$, but $\phi$ does not have to be proportional to $\log r$. In particular, $\phi$ can vanish asymptotically as $r \rightarrow \infty$, giving rise to an AdS planar black hole instead of a Lifshitz black hole. Analogous AdS planar black holes also arise from the Kaluza-Klein reduction of the theory discussed in section 2, as we commented under (A.2).

Note that the solution for the general cases contains only one integration constant, associated with the mass parameter $\mu$. The magnetic charge parameter $\alpha$ is fixed in terms of the "cosmological constant" (A.9). When $a=0$, the metric in the Einstein frame is asymptotic to a Lifshitz spacetime; for non-vanishing $a$, the metric is conformal to a Lifshitz spacetime, with a hyperscaling violation. To be explicit, we define a new radial coordinate

$$
\rho=r^{1+\frac{1}{2} \gamma a}
$$

in terms of which the metric becomes

$$
\begin{aligned}
d s^{2} & =\rho^{-\frac{2 \gamma a}{2+\gamma a}} d \tilde{s}^{2}=e^{-a \phi} d \tilde{s}^{2} \\
d \tilde{s}^{2} & =-\rho^{\frac{2\left(\gamma^{2}+(n-2) \gamma a\right)}{(n-2)(2+\gamma a)}} \tilde{f} d t^{2}+\frac{d \rho^{2}}{\tilde{f}}+\rho^{2} d \Sigma^{2}, \\
\tilde{f} & =\sigma\left(1+\frac{1}{2} \gamma a\right)^{2}\left[\rho^{2}-\mu \rho^{-\frac{2(n-2)(n-3)+\gamma^{2}}{(n-2)(2+\gamma a)}}\right] .
\end{aligned}
$$

We find that the transverse and traceless modes also satisfy the wave equation

$$
\left(\square-m(r)^{2}\right) \Psi(r, t)=0,
$$

but now with $m(r)^{2}$ given by

$$
m(r)^{2}=\nu \alpha^{2} r^{\gamma a} .
$$

It is clear that when $\gamma a \geq 0$, the mass is non-vanishing as $r \rightarrow \infty$. It follows that the zero-frequency mode $\chi$ cannot approach a constant as $r \rightarrow \infty$. In order for $\chi$ to approach 
1 asymptotically, as in the case we discussed in the main text, it is necessary to have $m(r)$ vanish on the boundary with a sufficiently rapid fall off. However, the solution (A.10) implies that we must have $\gamma a>-2$. This makes it impossible that $\chi$ can approach 1 as $r \rightarrow \infty$. The implications for the dual boundary theory in this case will be addressed in a future publication.

Open Access. This article is distributed under the terms of the Creative Commons Attribution License (CC-BY 4.0), which permits any use, distribution and reproduction in any medium, provided the original author(s) and source are credited.

\section{References}

[1] E. D'Hoker and P. Kraus, Magnetic brane solutions in AdS, JHEP 10 (2009) 088 [arXiv:0908.3875] [INSPIRE].

[2] T. Andrade and B. Withers, A simple holographic model of momentum relaxation, JHEP 05 (2014) 101 [arXiv:1311.5157] [INSPIRE].

[3] S.A. Hartnoll, D.M. Ramirez and J.E. Santos, Entropy production, viscosity bounds and bumpy black holes, JHEP 03 (2016) 170 [arXiv:1601.02757] [INSPIRE].

[4] J.M. Maldacena, The large- $N$ limit of superconformal field theories and supergravity, Int. J. Theor. Phys. 38 (1999) 1113 [Adv. Theor. Math. Phys. 2 (1998) 231] [hep-th/9711200] [INSPIRE].

[5] S.S. Gubser, I.R. Klebanov and A.M. Polyakov, Gauge theory correlators from noncritical string theory, Phys. Lett. B 428 (1998) 105 [hep-th/9802109] [InSPIRE].

[6] E. Witten, Anti-de Sitter space and holography, Adv. Theor. Math. Phys. 2 (1998) 253 [hep-th/9802150] [INSPIRE].

[7] O. Aharony, S.S. Gubser, J.M. Maldacena, H. Ooguri and Y. Oz, Large-N field theories, string theory and gravity, Phys. Rept. 323 (2000) 183 [hep-th/9905111] [INSPIRE].

[8] G. Policastro, D.T. Son and A.O. Starinets, The Shear viscosity of strongly coupled $N=4$ supersymmetric Yang-Mills plasma, Phys. Rev. Lett. 87 (2001) 081601 [hep-th/0104066] [INSPIRE].

[9] D.T. Son and A.O. Starinets, Minkowski space correlators in AdS/CFT correspondence: recipe and applications, JHEP 09 (2002) 042 [hep-th/0205051] [INSPIRE].

[10] P. Kovtun, D.T. Son and A.O. Starinets, Holography and hydrodynamics: diffusion on stretched horizons, JHEP 10 (2003) 064 [hep-th/0309213] [INSPIRE].

[11] P. Kovtun, D.T. Son and A.O. Starinets, Viscosity in strongly interacting quantum field theories from black hole physics, Phys. Rev. Lett. 94 (2005) 111601 [hep-th/0405231] [INSPIRE].

[12] H.-S. Liu, H. Lü and C.N. Pope, Generalized Smarr formula and the viscosity bound for Einstein-Maxwell-dilaton black holes, Phys. Rev. D 92 (2015) 064014 [arXiv:1507.02294] [INSPIRE].

[13] H.-S. Liu, Global scaling symmetry, Noether charge and universality of shear viscosity, Phys. Rev. D 93 (2016) 106001 [arXiv:1601.07875] [INSPIRE]. 
[14] Y. Kats and P. Petrov, Effect of curvature squared corrections in AdS on the viscosity of the dual gauge theory, JHEP 01 (2009) 044 [arXiv:0712.0743] [INSPIRE].

[15] M. Brigante, H. Liu, R.C. Myers, S. Shenker and S. Yaida, Viscosity bound violation in higher derivative gravity, Phys. Rev. D 77 (2008) 126006 [arXiv:0712.0805] [INSPIRE].

[16] R.-G. Cai, Z.-Y. Nie, N. Ohta and Y.-W. Sun, Shear viscosity from Gauss-Bonnet gravity with a dilaton coupling, Phys. Rev. D 79 (2009) 066004 [arXiv:0901.1421] [INSPIRE].

[17] S. Cremonini, The shear viscosity to entropy ratio: a status report, Mod. Phys. Lett. B 25 (2011) 1867 [arXiv: 1108.0677] [INSPIRE].

[18] M. Natsuume and M. Ohta, The shear viscosity of holographic superfluids, Prog. Theor. Phys. 124 (2010) 931 [arXiv: 1008.4142] [INSPIRE].

[19] A. Rebhan and D. Steineder, Violation of the holographic viscosity bound in a strongly coupled anisotropic plasma, Phys. Rev. Lett. 108 (2012) 021601 [arXiv:1110.6825] [INSPIRE].

[20] J. Erdmenger, P. Kerner and H. Zeller, Non-universal shear viscosity from Einstein gravity, Phys. Lett. B 699 (2011) 301 [arXiv:1011.5912] [INSPIRE].

[21] K.A. Mamo, Holographic RG flow of the shear viscosity to entropy density ratio in strongly coupled anisotropic plasma, JHEP 10 (2012) 070 [arXiv:1205.1797] [INSPIRE].

[22] R. Critelli, S.I. Finazzo, M. Zaniboni and J. Noronha, Anisotropic shear viscosity of a strongly coupled non-Abelian plasma from magnetic branes, Phys. Rev. D 90 (2014) 066006 [arXiv: 1406.6019] [INSPIRE].

[23] A. Bhattacharyya and D. Roychowdhury, Viscosity bound for anisotropic superfluids in higher derivative gravity, JHEP 03 (2015) 063 [arXiv:1410.3222] [INSPIRE].

[24] X.-H. Ge, Y. Ling, C. Niu and S.-J. Sin, Thermoelectric conductivities, shear viscosity and stability in an anisotropic linear axion model, Phys. Rev. D 92 (2015) 106005 [arXiv: 1412.8346] [INSPIRE].

[25] S. Jain, R. Samanta and S.P. Trivedi, The shear viscosity in anisotropic phases, JHEP 10 (2015) 028 [arXiv: 1506.01899] [INSPIRE].

[26] X.-H. Feng, H.-S. Liu, H. Lü and C.N. Pope, Black hole entropy and viscosity bound in Horndeski gravity, JHEP 11 (2015) 176 [arXiv:1509.07142] [INSPIRE].

[27] X.-H. Feng, H.-S. Liu, H. Lü and C.N. Pope, Thermodynamics of charged black holes in Einstein-Horndeski-Maxwell theory, Phys. Rev. D 93 (2016) 044030 [arXiv:1512.02659] [INSPIRE].

[28] L. Alberte, M. Baggioli and O. Pujolàs, Viscosity bound violation in holographic solids and the viscoelastic response, JHEP 07 (2016) 074 [arXiv: 1601.03384] [INSPIRE].

[29] P. Burikham and N. Poovuttikul, Shear viscosity in holography and effective theory of transport without translational symmetry, Phys. Rev. D 94 (2016) 106001 [arXiv: 1601.04624] [INSPIRE].

[30] Y. Bardoux, M.M. Caldarelli and C. Charmousis, Shaping black holes with free fields, JHEP 05 (2012) 054 [arXiv: 1202.4458] [inSPIRE].

[31] M. Taylor and W. Woodhead, Inhomogeneity simplified, Eur. Phys. J. C 74 (2014) 3176 [arXiv:1406.4870] [INSPIRE]. 
[32] M. Ortaggio, J. Podolsky and M. Zofka, Robinson-Trautman spacetimes with an electromagnetic field in higher dimensions, Class. Quant. Grav. 25 (2008) 025006 [arXiv:0708.4299] [INSPIRE].

[33] M. Ortaggio, J. Podolsky and M. Zofka, Static and radiating p-form black holes in the higher dimensional Robinson-Trautman class, JHEP 02 (2015) 045 [arXiv:1411.1943] [INSPIRE].

[34] A. Donos, J.P. Gauntlett and C. Pantelidou, Spatially modulated instabilities of magnetic black branes, JHEP 01 (2012) 061 [arXiv:1109.0471] [INSPIRE].

[35] M. Cvetič et al., Embedding AdS black holes in ten-dimensions and eleven-dimensions, Nucl. Phys. B 558 (1999) 96 [hep-th/9903214] [INSPIRE].

[36] R.A. Davison and B. Goutéraux, Momentum dissipation and effective theories of coherent and incoherent transport, JHEP 01 (2015) 039 [arXiv:1411.1062] [INSPIRE].

[37] A. Buchel and S. Cremonini, Viscosity bound and causality in superfluid plasma, JHEP 10 (2010) 026 [arXiv: 1007.2963] [INSPIRE].

[38] S. Kachru, X. Liu and M. Mulligan, Gravity duals of Lifshitz-like fixed points, Phys. Rev. D 78 (2008) 106005 [arXiv:0808.1725] [INSPIRE].

[39] M. Taylor, Non-relativistic holography, arXiv:0812.0530 [INSPIRE].

[40] C. Charmousis, B. Gouteraux, B.S. Kim, E. Kiritsis and R. Meyer, Effective holographic theories for low-temperature condensed matter systems, JHEP 11 (2010) 151 [arXiv: 1005.4690] [INSPIRE].

[41] N. Iizuka, N. Kundu, P. Narayan and S.P. Trivedi, Holographic Fermi and non-Fermi liquids with transitions in dilaton gravity, JHEP 01 (2012) 094 [arXiv:1105.1162] [INSPIRE].

[42] B. Gouteraux and E. Kiritsis, Generalized holographic quantum criticality at finite density, JHEP 12 (2011) 036 [arXiv:1107.2116] [INSPIRE].

[43] L. Huijse, S. Sachdev and B. Swingle, Hidden Fermi surfaces in compressible states of gauge-gravity duality, Phys. Rev. B 85 (2012) 035121 [arXiv:1112.0573] [INSPIRE]. 